\title{
A combined genotype of three SNPs in the bovine PPARD gene is related to growth performance in Chinese cattle
}

\section{Jieping Huang et al.}

Correspondence to: Yun Ma (mayun_666@126.com)

The copyright of individual parts of the supplement might differ from the CC BY 3.0 License. 
Table S1. Details of primers used for RT-PCR and SNP detection in bovine PPARD gene.

\begin{tabular}{|c|c|c|c|c|}
\hline Name & Primer sequence (5'-3') & Size (bp) & $\operatorname{Tm}\left({ }^{\circ} \mathrm{C}\right)$ & Used for \\
\hline Bovine-TUBAIA & $\begin{array}{l}\text { F:GGAGGTTCGCACTGGCAC } \\
\text { R:CGCCTTGCCAATGGTGTAG }\end{array}$ & 107 & 54 & RT-PCR for TUBAIA \\
\hline Bovine- $\beta$-actin & $\begin{array}{l}\text { F:CTGGGCGTAATGGTGGGC } \\
\text { R:CTGATGCCGTGCTCAATGG }\end{array}$ & 112 & 54 & RT-PCR for $\beta$-actin \\
\hline PPARD-mRNA & $\begin{array}{l}\text { F:GTGATCCACGACATCGAGACG } \\
\text { R:GCACTGGCAGCGGTAGAAG }\end{array}$ & 120 & 54 & RT-PCR for PPARD \\
\hline PPARB-P1 & $\begin{array}{l}\text { F:TCCTGTCTTCCCTTTCGTCC } \\
\text { R:GGAGACAACTCGCCCAAGAT }\end{array}$ & 427 & 59 & (nt71361-71787) Partial intron 1 and 2; exon 2 \\
\hline PPARB-P2 & $\begin{array}{l}\text { F:GAGACTGAACCTCGGAAAGG } \\
\text { R:TTCGCAGTGGATCTGGTCT }\end{array}$ & 582 & 56 & (nt79690-80271) Partial intron 2 and 3; exon 3 \\
\hline PPARB-P3 & $\begin{array}{l}\text { F:ATTGTTTTACTTTCCTTCTGCCTCT } \\
\text { R:GCTTTTCACACCTTGTCGGG }\end{array}$ & 645 & 60 & (nt80758-81402) Partial intron 3 and 4; exon 4 \\
\hline PPARB-P4 & $\begin{array}{l}\text { F:TAGACTCTGTGAACAAGATAGCCA } \\
\text { R:TGGCAGCGGTAGAAGACG }\end{array}$ & 670 & 57 & (nt82121-82790) Partial intron 4 and 5; exon 5 \\
\hline PPARB-P5 & $\begin{array}{l}\text { F:CCACACGGCGGTGAGTGTC } \\
\text { R:ATTGATACGTCTCCTTTTCCTTCT }\end{array}$ & 778 & 62 & (nt82491-83268) Partial intron 5 and 6; exon 6 \\
\hline PPARB-P6 & $\begin{array}{l}\text { F:CTGCCCATAGCCTTGTTAGT } \\
\text { R:GAGCCAGAAGCCGTGAGT }\end{array}$ & 554 & 55 & (nt83818-84361) Partial intron 6 and exon 7 \\
\hline PPARB-P7 & $\begin{array}{l}\text { F:TCATTTCACAAAGTGGGGGG } \\
\text { R:CAATGTCTTTTCTTGTGCAGCAG }\end{array}$ & 564 & 59 & nt(-2146)-(-1583) Partial promoter region \\
\hline PPARB-P8 & $\begin{array}{l}\text { F:TACTGAGACCATAGGCCACAATT } \\
\text { R:CTGCCGAACTACAGTCCGTG }\end{array}$ & 601 & 61 & nt(-1653)-(-1053) Partial promoter region \\
\hline PPARB-P9 & $\begin{array}{l}\text { F:CCCCATTTACAGGGCAAGTATATT } \\
\text { R:CCTGTTCATCGCCAATACCC }\end{array}$ & 624 & 58 & nt(-1186)-(-563) Partial promoter region \\
\hline
\end{tabular}


Table S2. Genetic characteristics of three SNPs detected in this study.

\begin{tabular}{|c|c|c|c|c|c|c|c|c|c|c|c|}
\hline \multirow{2}{*}{$\frac{\text { SNPs }}{\text { SNP1 }}$} & \multirow[t]{2}{*}{ Breeds } & \multirow[t]{2}{*}{ Sample } & \multicolumn{3}{|c|}{ Genotype frequencies } & \multicolumn{2}{|c|}{ Allele frequency } & \multirow[t]{2}{*}{ HWE-P value* } & \multirow[t]{2}{*}{ PIC } & \multirow[t]{2}{*}{$\mathbf{H}_{\mathbf{e}}$} & \multirow[t]{2}{*}{$\mathrm{Ne}$} \\
\hline & & & GG & GA & $\mathbf{A A}$ & G & $\mathbf{A}$ & & & & \\
\hline & NY & 139 & 0.043 & 0.942 & 0.015 & 0.043 & 0.942 & $106.582 * *$ & 0.375 & 0.500 & 1.998 \\
\hline & $\mathrm{JX}$ & 141 & 0.454 & 0.518 & 0.028 & 0.454 & 0.518 & $8.861^{*}$ & 0.325 & 0.410 & 1.694 \\
\hline & LX & 114 & 0.421 & 0.579 & 0.000 & 0.421 & 0.579 & $17.384 * *$ & 0.327 & 0.411 & 1.699 \\
\hline & $\mathrm{QC}$ & 30 & 0.700 & 0.300 & 0.000 & 0.700 & 0.300 & 0.141 & 0.223 & 0.255 & 1.342 \\
\hline & $\mathrm{BH}$ & 30 & 0.567 & 0.333 & 0.100 & 0.567 & 0.333 & 0.201 & 0.315 & 0.391 & 1.642 \\
\hline & GY & 60 & 0.067 & 0.933 & 0.000 & 0.067 & 0.933 & 43.339** & 0.374 & 0.498 & 1.991 \\
\hline \multirow[t]{7}{*}{ SNP 2} & & & $\mathbf{A A}$ & GA & GG & $\mathbf{A}$ & G & & & & \\
\hline & NY & 139 & 0.000 & 0.317 & 0.683 & 0.158 & 0.842 & 3.772 & 0.251 & 0.266 & 1.363 \\
\hline & $\mathrm{JX}$ & 141 & 0.000 & 0.539 & 0.461 & 0.270 & 0.730 & $17.719 * *$ & 0.316 & 0.394 & 1.650 \\
\hline & LX & 114 & 0.000 & 0.430 & 0.570 & 0.215 & 0.785 & $7.252 *$ & 0.281 & 0.337 & 1.509 \\
\hline & $\mathrm{QC}$ & 30 & 0.000 & 0.567 & 0.433 & 0.283 & 0.717 & 3.278 & 0.324 & 0.406 & 1.684 \\
\hline & $\mathrm{BH}$ & 30 & 0.000 & 0.700 & 0.300 & 0.350 & 0.650 & $6.976 *$ & 0.352 & 0.455 & 1.835 \\
\hline & GY & 60 & 0.000 & 0.133 & 0.867 & 0.067 & 0.933 & 0.205 & 0.117 & 0.124 & 1.142 \\
\hline \multirow[t]{7}{*}{ SNP 3} & & & TT & TC & $\mathrm{CC}$ & $\mathbf{T}$ & C & & & & \\
\hline & NY & 139 & 0.151 & 0.612 & 0.237 & 0.457 & 0.543 & 7.495* & 0.373 & 0.496 & 1.985 \\
\hline & $\mathrm{JX}$ & 141 & 0.291 & 0.667 & 0.042 & 0.624 & 0.376 & $24.977 * *$ & 0.359 & 0.469 & 1.884 \\
\hline & LX & 114 & 0.281 & 0.631 & 0.088 & 0.597 & 0.403 & $11.099 * *$ & 0.366 & 0.481 & 1.928 \\
\hline & $\mathrm{QC}$ & 30 & 0.500 & 0.367 & 0.133 & 0.683 & 0.317 & 0.267 & 0.339 & 0.433 & 1.763 \\
\hline & $\mathrm{BH}$ & 30 & 0.700 & 0.267 & 0.033 & 0.833 & 0.167 & 0.142 & 0.239 & 0.278 & 1.385 \\
\hline & GY & 60 & 0.033 & 0.450 & 0.517 & 0.258 & 0.742 & 1.169 & 0.310 & 0.383 & 1.621 \\
\hline
\end{tabular}

Not: SNP = Single Nucleotide Polymorphism; HWE = Hardy-Weinberg equilibrium; PIC = polymorphism information content; $\mathrm{H}_{\mathrm{e}}=$ heterozygosity; $\mathrm{N}_{\mathrm{e}}=$ effective allele numbers. JX $=$ Jiaxian; $\mathrm{LX}=$ Luxi; $\mathrm{NY}=$ Nanyang; $\mathrm{QC}=$ Qinchuan; $\mathrm{BH}=$ Bohai; $\mathrm{GY}=$ Gaoyuan. $\chi^{2} 0.05=5.99, \chi^{2} 0.01=9.21$, * means the difference is significant at the 0.05 level. ** means the difference is significant at the 0.01 level. $\chi^{2}>5.99$ means deviating from Hardy-Weinberg equilibrium (HWE) with significant level. $\chi^{2}>9.21$ means deviating from HWE with highly significant level. PIC $>0.5$ means high diversity, $0.25<$ PIC $<0.5$ means moderate diversity, PIC $<0.25$ means low diversity. 
Table S3. Association analysis between SNPs loci of PPARD and growth traits in Henan cattle.

\begin{tabular}{|c|c|c|c|c|c|c|c|c|c|}
\hline \multirow{2}{*}{$\begin{array}{l}\text { Age } \\
\text { (month) }\end{array}$} & \multirow{2}{*}{$\begin{array}{l}\text { Growth } \\
\text { trait }\end{array}$} & \multicolumn{3}{|l|}{ SNP 1 } & \multicolumn{2}{|l|}{ SNP 2} & \multicolumn{3}{|l|}{ SNP 3} \\
\hline & & GG (24) & GA (147) & $\mathbf{A A}(2)$ & AG (85) & GG (88) & TT (37) & TC (108) & CC (28) \\
\hline 0 & WB (kg) & $\begin{array}{l}27.921 \pm \\
0.754^{\mathrm{Bb}}\end{array}$ & $\begin{array}{l}30.219 \pm \\
0.305^{\mathrm{A}}\end{array}$ & $\begin{array}{l}33.750 \pm \\
2.613^{a}\end{array}$ & $\begin{array}{l}29.107 \pm \\
0.402^{B}\end{array}$ & $\begin{array}{l}30.747 \pm \\
0.395^{A}\end{array}$ & $\begin{array}{l}28.608 \pm \\
0.615^{b}\end{array}$ & $\begin{array}{l}30.269 \pm \\
0.360^{\mathrm{a}}\end{array}$ & $\begin{array}{l}30.439 \pm \\
0.706^{a}\end{array}$ \\
\hline \multirow[t]{6}{*}{6} & $\mathrm{BW}(\mathrm{kg})$ & $\begin{array}{l}174.875 \pm \\
4.884^{\mathrm{a}}\end{array}$ & $\begin{array}{l}161.626 \pm \\
1.974^{b}\end{array}$ & $\begin{array}{l}158.250 \pm \\
16.920\end{array}$ & $\begin{array}{l}169.741 \pm \\
2.548^{\mathrm{A}}\end{array}$ & $\begin{array}{l}157.324 \pm \\
2.504^{B}\end{array}$ & $\begin{array}{l}162.730 \pm \\
3.992\end{array}$ & $\begin{array}{l}164.824 \pm \\
2.336\end{array}$ & $\begin{array}{l}158.946 \pm \\
4.589\end{array}$ \\
\hline & $\mathrm{BH}(\mathrm{cm})$ & $\begin{array}{l}101.583 \pm \\
2.482\end{array}$ & $\begin{array}{l}99.925 \pm \\
1.003\end{array}$ & $\begin{array}{l}108.000 \pm \\
8.596\end{array}$ & $\begin{array}{l}98.235 \pm \\
1.302^{\mathrm{b}}\end{array}$ & $\begin{array}{l}102.193 \pm \\
1.279^{\mathrm{a}}\end{array}$ & $\begin{array}{l}95.324 \pm \\
1.956^{b}\end{array}$ & $\begin{array}{l}101.185 \pm \\
1.145^{\mathrm{a}}\end{array}$ & $\begin{array}{l}103.143 \pm \\
2.249^{\mathrm{a}}\end{array}$ \\
\hline & $\mathrm{BL}(\mathrm{cm})$ & $\begin{array}{l}108.792 \pm \\
1.352\end{array}$ & $\begin{array}{l}106.687 \pm \\
0.546\end{array}$ & $\begin{array}{l}110.500 \pm \\
4.684\end{array}$ & $\begin{array}{l}108.306 \pm \\
0.709^{\mathrm{a}}\end{array}$ & $\begin{array}{l}105.784 \pm \\
0.697^{\mathrm{b}}\end{array}$ & $\begin{array}{l}110.135 \pm \\
1.063^{\mathrm{A}}\end{array}$ & $\begin{array}{l}106.296 \pm \\
0.622^{\mathrm{B}}\end{array}$ & $\begin{array}{l}105.714 \pm \\
1.222^{\mathrm{B}}\end{array}$ \\
\hline & $\mathrm{HG}(\mathrm{cm})$ & $\begin{array}{l}132.208 \pm \\
2.060^{\mathrm{a}}\end{array}$ & $\begin{array}{l}127.180 \pm \\
\mathbf{0 . 8 3 2}^{b}\end{array}$ & $\begin{array}{l}126.500 \pm \\
7.135\end{array}$ & $\begin{array}{l}129.035 \pm \\
1.101\end{array}$ & $\begin{array}{l}126.744 \pm \\
1.082\end{array}$ & $\begin{array}{l}129.176 \pm \\
1.678\end{array}$ & $\begin{array}{l}127.769 \pm \\
0.982\end{array}$ & $\begin{array}{l}126.536 \pm \\
1.929\end{array}$ \\
\hline & $\mathrm{HW}(\mathrm{cm})$ & $\begin{array}{l}12.917 \pm \\
0.872^{\mathrm{Bb}}\end{array}$ & $\begin{array}{l}16.374 \pm \\
0.352^{\mathrm{A}}\end{array}$ & $\begin{array}{l}19.750 \pm \\
3.021^{\mathrm{a}}\end{array}$ & $\begin{array}{l}13.835 \pm \\
0.426^{\mathrm{B}}\end{array}$ & $\begin{array}{l}17.960 \pm \\
0.419^{\mathrm{A}}\end{array}$ & $\begin{array}{l}14.176 \pm \\
0.692^{C}\end{array}$ & $\begin{array}{l}15.764 \pm \\
0.405^{B}\end{array}$ & $\begin{array}{l}18.911 \pm \\
0^{0.796}\end{array}$ \\
\hline & $\mathrm{ADG}(\mathrm{kg})$ & $\begin{array}{l}0.816 \pm \\
0.027^{\mathrm{A}}\end{array}$ & $\begin{array}{l}0.726 \pm \\
0.011^{B}\end{array}$ & $\begin{array}{l}0.715 \pm \\
0.093\end{array}$ & $\begin{array}{l}0.780 \pm \\
0.014^{\mathrm{A}}\end{array}$ & $\begin{array}{l}0.698 \pm \\
0.014^{\mathrm{B}}\end{array}$ & $\begin{array}{l}0.744 \pm \\
0.022\end{array}$ & $\begin{array}{l}0.745 \pm \\
0.013\end{array}$ & $\begin{array}{l}0.705 \pm \\
0.025\end{array}$ \\
\hline \multirow[t]{6}{*}{12} & BW (kg) & $\begin{array}{l}247.000 \pm \\
5.190\end{array}$ & $\begin{array}{l}239.929 \pm \\
2.097\end{array}$ & $\begin{array}{l}217.000 \pm \\
17.980\end{array}$ & $\begin{array}{l}240.141 \pm \\
2.776\end{array}$ & $\begin{array}{l}241.131 \pm \\
2.729\end{array}$ & $\begin{array}{l}240.784 \pm \\
4.214\end{array}$ & $\begin{array}{l}241.444 \pm \\
2.467\end{array}$ & $\begin{array}{l}237.375 \pm \\
4.844\end{array}$ \\
\hline & $\mathrm{BH}(\mathrm{cm})$ & $\begin{array}{l}113.958 \pm \\
0.775\end{array}$ & $\begin{array}{l}113.391 \pm \\
0.313\end{array}$ & $\begin{array}{l}115.000 \pm \\
2.684\end{array}$ & $\begin{array}{l}113.329 \pm \\
0.411\end{array}$ & $\begin{array}{l}113.642 \pm \\
0.404\end{array}$ & $\begin{array}{l}113.203 \pm \\
0.624\end{array}$ & $\begin{array}{l}113.435 \pm \\
0.365\end{array}$ & $\begin{array}{l}114.071 \pm \\
0.717\end{array}$ \\
\hline & $\mathrm{BL}(\mathrm{cm})$ & $\begin{array}{l}118.583 \pm \\
1.046\end{array}$ & $\begin{array}{l}116.952 \pm \\
0.423\end{array}$ & $\begin{array}{l}116.500 \pm \\
3.622\end{array}$ & $\begin{array}{l}117.306 \pm \\
0.557\end{array}$ & $\begin{array}{l}117.045 \pm \\
0.548\end{array}$ & $\begin{array}{l}118.892 \pm \\
0.834^{\mathrm{a}}\end{array}$ & $\begin{array}{l}116.583 \pm \\
0.488^{b}\end{array}$ & $\begin{array}{l}117.179 \pm \\
0.958\end{array}$ \\
\hline & $\mathrm{HG}(\mathrm{cm})$ & $\begin{array}{l}145.917 \pm \\
1.483\end{array}$ & $\begin{array}{l}143.194 \pm \\
0.599\end{array}$ & $\begin{array}{l}140.500 \pm \\
5.137\end{array}$ & $\begin{array}{l}145.129 \pm \\
0.775\end{array}$ & $\begin{array}{l}142.006 \pm \\
0.761\end{array}$ & $\begin{array}{l}147.243 \pm \\
1.162^{\mathrm{Aa}}\end{array}$ & $\begin{array}{l}142.514 \pm \\
0.68^{\mathrm{B}}\end{array}$ & $\begin{array}{l}142.607 \pm \\
1.336^{b}\end{array}$ \\
\hline & $\mathrm{HW}(\mathrm{cm})$ & $\begin{array}{l}15.958 \pm \\
0.846^{B}\end{array}$ & $\begin{array}{l}18.840 \pm \\
0.342^{\mathrm{A}}\end{array}$ & $\begin{array}{l}21.000 \pm \\
2.931\end{array}$ & $\begin{array}{l}16.641 \pm \\
0.419^{B}\end{array}$ & $\begin{array}{l}20.227 \pm \\
0.412^{\mathrm{A}}\end{array}$ & $\begin{array}{l}16.486 \pm \\
0.666^{\mathrm{C}}\end{array}$ & $\begin{array}{l}18.509 \pm \\
0.390^{B}\end{array}$ & $\begin{array}{l}20.911 \pm \\
0.766^{\mathrm{A}}\end{array}$ \\
\hline & ADG $(\mathrm{kg})$ & $\begin{array}{l}0.401 \pm \\
0.032\end{array}$ & $\begin{array}{l}0.435 \pm \\
0.013\end{array}$ & $\begin{array}{l}0.326 \pm \\
0.112\end{array}$ & $\begin{array}{l}0.391 \pm \\
0.017^{\mathrm{B}}\end{array}$ & $\begin{array}{l}0.466 \pm \\
0.016^{\mathrm{A}}\end{array}$ & $\begin{array}{l}0.434 \pm \\
0.026\end{array}$ & $\begin{array}{l}0.426 \pm \\
0.015\end{array}$ & $\begin{array}{l}0.436 \pm \\
0.030\end{array}$ \\
\hline
\end{tabular}




\begin{tabular}{|c|c|c|c|c|c|c|c|c|c|}
\hline \multirow[t]{6}{*}{18} & BW (kg) & $\begin{array}{l}294.458 \pm \\
6.357\end{array}$ & $\begin{array}{l}305.020 \pm \\
2.569\end{array}$ & $\begin{array}{l}335.000 \pm \\
22.022\end{array}$ & $\begin{array}{l}301.047 \pm \\
3.398\end{array}$ & $\begin{array}{l}306.659 \pm \\
3.339\end{array}$ & $\begin{array}{l}303.703 \pm \\
5.168\end{array}$ & $\begin{array}{l}302.472 \pm \\
3.025\end{array}$ & $\begin{array}{l}309.679 \pm \\
5.941\end{array}$ \\
\hline & $\mathrm{BH}(\mathrm{cm})$ & $\begin{array}{l}119.833 \pm \\
0.697\end{array}$ & $\begin{array}{l}120.724 \pm \\
0.282\end{array}$ & $\begin{array}{l}124.000 \pm \\
2.416\end{array}$ & $\begin{array}{l}119.894 \pm \\
0.364^{\mathrm{B}}\end{array}$ & $\begin{array}{l}121.358 \pm \\
0.358^{\mathrm{A}}\end{array}$ & $\begin{array}{l}120.541 \pm \\
0.565\end{array}$ & $\begin{array}{l}120.509 \pm \\
0.331\end{array}$ & $\begin{array}{l}121.268 \pm \\
0.650\end{array}$ \\
\hline & $\mathrm{BL}(\mathrm{cm})$ & $\begin{array}{l}125.500 \pm \\
1.084\end{array}$ & $\begin{array}{l}127.371 \pm \\
0.438\end{array}$ & $\begin{array}{l}131.000 \pm \\
3.754\end{array}$ & $\begin{array}{l}127.206 \pm \\
0.580\end{array}$ & $\begin{array}{l}127.102 \pm \\
0.570\end{array}$ & $\begin{array}{l}127.351 \pm \\
0.876\end{array}$ & $\begin{array}{l}126.755 \pm \\
0.513\end{array}$ & $\begin{array}{l}128.429 \pm \\
1.007\end{array}$ \\
\hline & $\mathrm{HG}(\mathrm{cm})$ & $\begin{array}{l}157.917 \pm \\
1.649\end{array}$ & $\begin{array}{l}159.412 \pm \\
0.666\end{array}$ & $\begin{array}{l}154.000 \pm \\
5.711\end{array}$ & $\begin{array}{l}158.865 \pm \\
0.877\end{array}$ & $\begin{array}{l}159.409 \pm \\
0.862\end{array}$ & $\begin{array}{l}159.892 \pm \\
1.309\end{array}$ & $\begin{array}{l}158.083 \pm \\
0.766^{b}\end{array}$ & $\begin{array}{l}162.232 \pm \\
1.505^{\mathrm{a}}\end{array}$ \\
\hline & HW (cm) & $\begin{array}{l}19.458 \pm \\
0.792^{\mathrm{B}}\end{array}$ & $\begin{array}{l}21.946 \pm \\
0.320^{\mathrm{A}}\end{array}$ & $\begin{array}{l}23.500 \pm \\
2.743\end{array}$ & $\begin{array}{l}20.341 \pm \\
0.408^{B}\end{array}$ & $\begin{array}{l}22.852 \pm \\
0.401^{\mathrm{A}}\end{array}$ & $\begin{array}{l}19.824 \pm \\
0.620^{c}\end{array}$ & $\begin{array}{l}21.625 \pm \\
0^{0.363^{b}}\end{array}$ & $\begin{array}{l}23.964 \pm \\
\text { 0.713 }^{\mathrm{a}}\end{array}$ \\
\hline & $\mathrm{ADG}(\mathrm{kg})$ & $\begin{array}{l}0.264 \pm \\
0.029^{B}\end{array}$ & $\begin{array}{l}0.362 \pm \\
0.012^{\mathrm{A}}\end{array}$ & $\begin{array}{l}0.656 \pm \\
0.100^{\mathrm{C}}\end{array}$ & $\begin{array}{l}0.338 \pm \\
0.016\end{array}$ & $\begin{array}{l}0.364 \pm \\
0.016\end{array}$ & $\begin{array}{l}0.350 \pm \\
0.024\end{array}$ & $\begin{array}{l}0.339 \pm \\
0.014^{b}\end{array}$ & $\begin{array}{l}0.402 \pm \\
0.028^{\mathrm{a}}\end{array}$ \\
\hline \multirow[t]{6}{*}{24} & BW (kg) & $\begin{array}{l}347.417 \pm \\
8^{8.677^{b}}\end{array}$ & $\begin{array}{l}367.500 \pm \\
3.506^{\mathrm{a}}\end{array}$ & $\begin{array}{l}410.000 \pm \\
30.059^{\mathrm{a}}\end{array}$ & $\begin{array}{l}358.612 \pm \\
4^{.635^{b}}\end{array}$ & $\begin{array}{l}\text { 371.574 } \pm \\
4.556^{\mathrm{a}}\end{array}$ & $\begin{array}{l}363.405 \pm \\
7.080\end{array}$ & $\begin{array}{l}362.894 \pm \\
4.144\end{array}$ & $\begin{array}{l}376.500 \pm \\
8.139\end{array}$ \\
\hline & $\mathrm{BH}(\mathrm{cm})$ & $\begin{array}{l}124.833 \pm \\
0.788\end{array}$ & $\begin{array}{l}126.133 \pm \\
0.318\end{array}$ & $\begin{array}{l}127.500 \pm \\
2.729\end{array}$ & $\begin{array}{l}125.212 \pm \\
0^{0.413}{ }^{b}\end{array}$ & $\begin{array}{l}126.699 \pm \\
0.406^{\mathrm{a}}\end{array}$ & $\begin{array}{l}125.473 \pm \\
0.633\end{array}$ & $\begin{array}{l}125.824 \pm \\
0.370\end{array}$ & $\begin{array}{l}127.179 \pm \\
0.728\end{array}$ \\
\hline & $\mathrm{BL}(\mathrm{cm})$ & $\begin{array}{l}136.167 \pm \\
1.307\end{array}$ & $\begin{array}{l}136.486 \pm \\
0.528\end{array}$ & $\begin{array}{l}136.500 \pm \\
4.529\end{array}$ & $\begin{array}{l}137.165 \pm \\
0.688\end{array}$ & $\begin{array}{l}135.744 \pm \\
0.677\end{array}$ & $\begin{array}{l}136.838 \pm \\
1.043\end{array}$ & $\begin{array}{l}135.847 \pm \\
0.611\end{array}$ & $\begin{array}{l}138.214 \pm \\
1.199\end{array}$ \\
\hline & $\mathrm{HG}(\mathrm{cm})$ & $\begin{array}{l}166.208 \pm \\
1.664^{b}\end{array}$ & $\begin{array}{l}170.724 \pm \\
0.672^{\mathrm{a}}\end{array}$ & $\begin{array}{l}167.500 \pm \\
5.763\end{array}$ & $\begin{array}{l}168.941 \pm \\
0.890\end{array}$ & $\begin{array}{l}171.142 \pm \\
0.875\end{array}$ & $\begin{array}{l}168.919 \pm \\
1.327^{\mathrm{B}}\end{array}$ & $\begin{array}{l}169.319 \pm \\
0.777^{\mathrm{B}}\end{array}$ & $\begin{array}{l}174.429 \\
1.526^{\mathrm{A}}\end{array}$ \\
\hline & HW (cm) & $\begin{array}{l}21.875 \pm \\
0.791^{B}\end{array}$ & $\begin{array}{l}24.524 \pm \\
0.320^{\mathrm{A}}\end{array}$ & $\begin{array}{l}26.250 \pm \\
2.741\end{array}$ & $\begin{array}{l}23.047 \pm \\
0.414^{B}\end{array}$ & $\begin{array}{l}25.267 \pm \\
0.407^{\mathrm{A}}\end{array}$ & $\begin{array}{l}22.676 \pm \\
0.635^{\mathrm{Cc}}\end{array}$ & $\begin{array}{l}24.236 \pm \\
0.372^{b}\end{array}$ & $\begin{array}{l}25.929 \pm \\
0.730^{\text {Aa }}\end{array}$ \\
\hline & $\mathrm{ADG}(\mathrm{kg})$ & $\begin{array}{l}0.294 \pm \\
0.033\end{array}$ & $\begin{array}{l}0.347 \pm \\
0.013\end{array}$ & $\begin{array}{l}0.417 \pm \\
0.114\end{array}$ & $\begin{array}{l}0.320 \pm \\
0.017\end{array}$ & $\begin{array}{l}0.361 \pm \\
0.017\end{array}$ & $\begin{array}{l}0.332 \pm \\
0.027\end{array}$ & $\begin{array}{l}0.336 \pm \\
0.016\end{array}$ & $\begin{array}{l}0.371 \pm \\
0.031\end{array}$ \\
\hline
\end{tabular}

\footnotetext{
Notes: capital letter means differ of the value at $P<0.01$; lowercase letter means differ of the value at $P<0.05$. WB $=$ weight of birth; $\mathrm{BW}=$ body weight; $\mathrm{BH}$ = body height; $\mathrm{BL}=$ body length $\mathrm{HG}=$ heart girth; $\mathrm{HW}=$ hip width; $\mathrm{ADG}=$ average daily gain.
} 